\title{
Body Building Music: The Kinase installation
}

\author{
MONNAZZI, João Paulo Silva ${ }^{l}$, FARIA, Regis Rossi Alves ${ }^{2}$ \\ ${ }^{l}$ Escola de Comunicação e Artes da Universidade de São Paulo Av. \\ Prof. Luciano Gualberto, 158, tv,3 - 05508-900 São Paulo, SP. \\ ${ }^{2}$ Escola de Artes, Ciências e Humanidades, Universidade de São \\ Paulo Rua Arlindo Bettio, 1000 - 03828-000, São Paulo, SP \\ joao.monnazzi@usp.br, regis@usp.br
}

\section{Extend Abstract}

Thinking on the congruencies between music and sports, we propose with this art installation some novel paths and connections for music production in a little explored field, in the interdisciplinarity with sports.

Some similarities in the acting of musicians and athletes, such as the need of technical domain through discipline and practice. A musician who wants to develop her/his technical skills needs to follow a hard routine of practical studies, focusing in improving motor abilities with the proposing to play the piece in the better way possible.

This process has a close proximity with the athlete's during their preparation. Hours of intense practice to improve some motor skills that can enable them to improve their performance. The disciplines can be interpolated in a way that we can argue: there is always something physical on a music interpretation, as well as there is always something artistic in a sport competition.

In the inner area between art/music and sports some modalities are easier to verify this symbiosis, as in the choreographic sports. These modalities are evaluated by both physical and artistic parameters. Our work focus in a particular sport modality that has a part of scoring which is evaluated through a choreographic routine: The Bodybuilding.

\section{The sport and the Body Building Music engine}

Bodybuilding is a sportive modality evaluated using artistic parameters [1]. The body of athletes are analyzed by symmetry, conditioning and volume of muscle mass. The competitor who has the most symmetric, lean fat free and body muscle, wins. During the bodybuilding competition, one of the most expected moment is the solo presentation, when the bodybuilder performs a choreographic routine with musical accompaniment. In this part of the championship, the evaluated parameters are creativity and synchronicity. Our interactive system supports this part of the show.

Body Building Music (BBM) is an interactive music system that allows the user to control a musical performance and its parameters through gestures derived from the bodybuilding sport. The system was enabled by musical controllers that use Kinect (camera sensor based) as a motion sensor to trigger or reshuffle sound materials [2].

The idea of the system is to provide the user with a double experience. The first is to compose music in real time in a way not requiring a prior knowledge of musical knowledge, and the second is to experience and get to know the bodybuilding sport through the poses required in the official championships.

The system was structured in functional levels and implemented with a mixed computer programming strategy, using Pure Data (Pd) and C-based routines that permit to integrate its physical components, comprised of a gestural sensor and a computer responsible for gestural processing and music generation. The functional rationale is attached to the gestural poses from the bodybuilding sport [3].

The mapping strategy used was a gesture for each sound (one-to-one mapping) [4]. Eight mandatory poses from bodybuilding championship were chosen to control inputs and output of musical layers and two continuous gestures were programmed to control a EQ filter and the dynamics of the musical sounds played back. We can then separate the control process in three levels, as below.

First level: the music structure interactive control level. In this level we can control the input or output of musical layers previously recorded. Each one of the 8 poses of bodybuilding is to deliver a different musical material. By posing, the user is enabled to mount a musical piece through sequencing of poses matched to musical parts. The order in which the poses are made will affect or to change the musical structure.

Second level: the effect (fx) control level. This level can be actived any time during the process by hanging the right hand open for more than two seconds. After this movement the user is enabled then to control the central frequency of a semi-parametric filter affecting the sound equalization. Through a continuous movement of his/her mirrored hands, the user can close or open the spectral coverage of the filter.

Third level: the dynamic level control. This control level is activated by hanging the left hand open 
for more than two seconds. After accessing this control stage, the user can modify the dynamics of all active playing musical parts. By raising both hands, the dynamic level increases, as well as moving both hands down will make the volume to decrease.

These three control levels were implemented to enable the user to experience a real time composition and music modulation process through a non-musical classical gesture, and he/she can still learn about bodybuilding and try some of its poses, experiencing the particularities of that sport.

The hardware system is composed by a camera sensor (Kinect), a laptop, a DAW (digital audio workstation) and a sound monitoring system (see fig. 1). It captures the performer gestures that will be mapped accordingly to a table of poses.

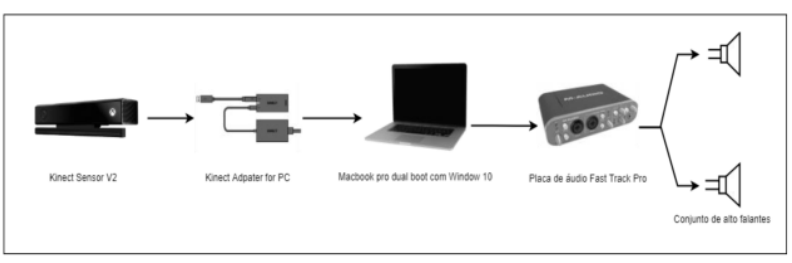

Figure 1: Hardware connections diagram

The user's movements are captured by the Kinect, compared with a repository of poses previously built. If the program recognizes a valid movement it releases an OSC message to a Pure Data (Pd) patch, where the musical generative actions are programmed, synthesizing the audio and delivering it using a stereo sound system. Figure 2 below shows a flowchart of the processes integrated in the system.

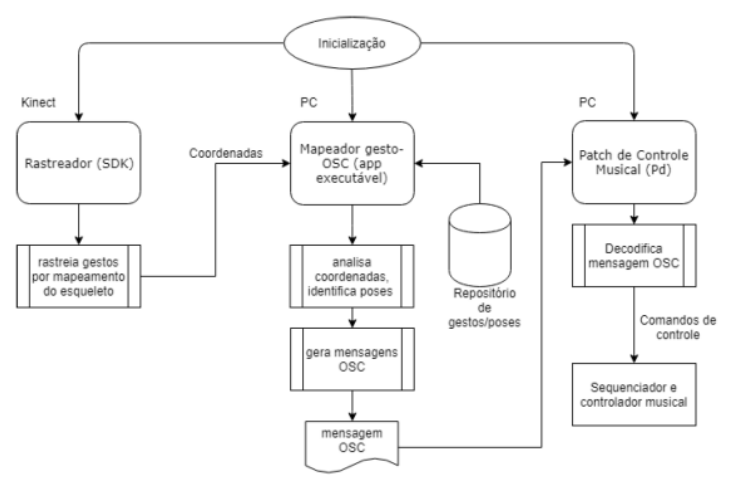

Figure 2: BBM system flowchart \

\section{The Kinase musical creation}

For the system to function as intended, bridging with musical real-time creation, it was composed an interactive musical piece [5], that would permit to use all the BBM system function. This was how Kinase was born, as a musical interactive composition-based musical creation.

Kinase is a modular piece, layer-based, which is instantiated dynamically through a real-time generation of audio controlled by gestures. During the system usage, the user can add just one of the eight available musical layers at a time, and it thus will be overlapped with other layers obeying a given synchronicity, so as to permit that musical parts with different execution times are triggered in the beginning of the right measures.

Figure 3 shows the piece structure and the instrumentation. The input order of each layer defines the music structure as well as the moment of activation of each layer. Other possible actions include the activation and control of a filter that will act on all activated tracks, serving as an artistic resource for performance. In the same way, the user can activate and control the dynamics of the piece through continuous gestures.

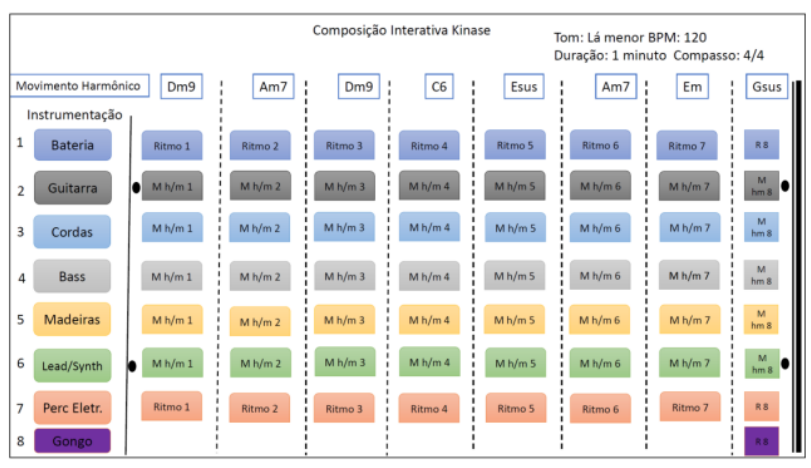

Figure 3: The Kinase structure

\section{Final remarks}

The installation is proposed to be run during the conference, in a public place, and be available for people interested in trying up with the system. A table describing the poses that control the creative process will be available, as well as some guidance to train users on how to use it.

\section{References}

[1] A. Locks and N. Richardosn. Critical Reading in Bodybuilding. Routledge, Nova York, 2012.

[2] MONNAZZI. J. and FARIA. R. O uso do Kinect como instrumento digital em sistemas interativos para performance. $15^{\circ}$ Annals Congressional of Audio and Engineering Society of Brazil AES.

[3] MONNAZZI. J. and FARIA, R. Body Building Music: Sistema interativo para criação musical controlado por performance coreográfica esportiva. Master's dissertation. São Paulo, 2018.

[4] J. Rova, M. Wanderley, S. Dubnov and P. Depalle. Instrumental Gestural Mapping Strategies as Expressivity Determinants in Computer Music Performance. In: Proceedings of the AIMI International Workshop, Genova, Associazione di Informatica Musicale Italiana, pages $68-73,1997$.

[5] M. Sweet. Writing Interactive Music for Video Games. Pearson Education. Crawfordsville, 2015. 